\title{
Enhancing the production of cephalosporin $C$ through modulating the autophagic process of Acremonium chrysogenum
}

Honghua $\mathrm{Li}^{1,2+}{ }^{\text {, Pengjie Hu}}{ }^{1 \dagger}$, Ying Wang ${ }^{1 \dagger}$, Yuanyuan Pan ${ }^{1}$ and Gang Liu ${ }^{1,2^{*}}$ (D)

\begin{abstract}
Background: Autophagy is used for degradation of cellular components and nutrient recycling. Atg8 is one of the core proteins in autophagy and used as a marker for autophagic detection. However, the autophagy of filamentous fungi is poorly understood compared with that of Saccharomyces cerevisiae. Our previous study revealed that disruption of the autophagy related gene Acatg1 significantly enhanced cephalosporin C yield through reducing degradation of cephalosporin biosynthetic proteins in Acremonium chrysogenum, suggesting that modulation of autophagic process is one promising way to increase antibiotic production in A. chrysogenum.
\end{abstract}

Results: In this study, a S. cerevisiae ATG8 homologue gene Acatg8 was identified from A. chrysogenum. Acatg8 could complement the ATG8 mutation in S. cerevisiae, indicating that Acatg 8 is a functional homologue of ATG8. Microscope observation demonstrated the fluorescently labeled AcAtg 8 was localized in the cytoplasm and autophagosome of $A$. chrysogenum, and the expression of Acatg 8 was induced by nutrient starvation. Gene disruption and genetic complementation revealed that Acatg8 is essential for autophagosome formation. Disruption of Acatg8 significantly reduced fungal conidiation and delayed conidial germination. Localization of GFP-AcAtg8 implied that autophagy is involved in the early phase of conidial germination. Similar to Acatg 1, disruption of Acatg8 remarkably enhanced cephalosporin $C$ yield. The cephalosporin $C$ biosynthetic enzymes (isopenicillin N synthase PcbC and isopenicillin $\mathrm{N}$ epimerase CefD2) and peroxisomes were accumulated in the Acatg8 disruption mutant ( $\triangle$ Acatg8), which might be the main reasons for the enhancement of cephalosporin C production. However, the biomass of $\triangle A$ catg 8 decreased drastically at the late stage of fermentation, suggesting that autophagy is critical for A. chrysogenum cell survival under nutrition deprived condition. Disruption of Acatg8 also resulted in accumulation of mitochondria, which might produce more reactive oxygen species (ROS) which promotes fungal death. However, the premature death is unfavorable for cephalosporin C production. To solve this problem, a plasmid containing Acatg8 under control of the xylose/xylaninducible promoter was introduced into $\triangle$ Acatg8. Conidiation and growth of the recombinant strain restored to the wild-type level in the medium supplemented with xylose, while the cephalosporin C production maintained at a high level even prolonged fermentation.

Conclusions: Our results demonstrated inducible expression of Acatg8 and disruption of Acatg 8 remarkably increased cephalosporin $\mathrm{C}$ production. This study provides a promising approach for yield improvement of cephalosporin C in A. chrysogenum.

\footnotetext{
*Correspondence: liug@im.ac.cn

${ }^{\dagger}$ Honghua Li, Pengjie Hu and Ying Wang Contributed equally to this work

1 State Key Laboratory of Mycology, Institute of Microbiology, Chinese

Academy of Sciences, Beijing 100101, China

Full list of author information is available at the end of the article
} 
Keywords: Acatg8, Acremonium chrysogenum, Autophagy, Cephalosporin C, Conidial germination

\section{Background}

Macroautophagy (hereafter autophagy) is generally used for degradation of cellular components and nutrient recycling in eukaryotes [1,2]. The degradation of cytoplasm components (such as cytosol, macromolecular complexes, and organelles) takes place in vacuole/ lysosome of eukaryotes. Autophagy normally occurs at a low constitutive level, but it is up-regulated under nutrient starvation condition. Autophagy begins with the formation of a sequestering membrane termed phagophore, and the phagophore expands and forms a double-membrane-bound vesicle known as autophagosome. The autophagosomes enter the vacuoles through membrane fusing and form autophagic bodies in which autophagic cargoes are degraded. As one of the core proteins, the ubiquitin-like protein Atg8 is essential for autophagosome formation and highly conserved in eukaryotes. Besides, Atg8 is also used as a biological marker for autophagic detection [3, 4].

In mammals, autophagy is involved in carcinogenesis, neurodegenerative diseases and developmental processes [5, 6]. Autophagy has been extensively studied in Saccharomyces cerevisiae, where more than 40 genes are involved in this process. In S. cerevisiae, autophagy deficient mutants not only show a reduction of cell viability under starvation condition, but also display defect in sporulation [7]. Recently, autophagy of filamentous fungi has received more attention since it is involved in fungal survival, reproduction and pathogenicity [8]. In Magnaporthe grisea, deletion of the autophagy related genes results in reduction of conidiation and non-pathogenic phenotype [4]. In Fusarium graminearum, autophagy is important for lipid turnover, deoxynivalenol production and infection in plant [9]. In the cucumber anthracnose fungus Colletotrichum orbiculare, autophagy is required for host invasion [10]. In the well-established aging model Podospora anserine, autophagy is related with aging and life span as a longevity-assurance mechanism [11]. In Aspergillus ory$z a e$, autophagy is required for the formation of aerial hyphae and conidia [12], and deficiency of autophagy enhances the production of bovine chymosin [13]. In Sordaria macrospora, autophagy is used to sustain high energy levels for mycelia growth and morphological differentiation [14]. In Penicillium chrysogenum, the atg1 deletion mutant showed onefold increase in penicillin production [15]. Thus, autophagy is extensively related with morphological differentiation and secondary metabolite productions in filamentous fungi.
Acremonium chrysogenum is well known for producing the pharmaceutically relevant $\beta$-lactam antibiotic cephalosporin $\mathrm{C}$ (CPC). The cephalosporin biosynthetic genes of $A$. chrysogenum are localized in two separated clusters [16]. The CPC biosynthetic pathway has been well studied and at least 6 biosynthetic genes $(p c b A B, p c b C$, $c e f D 1$, cefD2, cefEF and cefG) are essential for the CPC biosynthesis $[17,18]$. Like most of secondary metabolites, cephalosporin $\mathrm{C}$ is produced at the anaphase of exponential growth and stationary phase $[19,20]$. During this time, the nutrients are depleted and autophagy is induced. It is speculated that the CPC biosynthetic proteins including PcbAB, PcbC, CefD1 and CefD2 could be degraded through autophagic process. In fact, disruption of an autophagy-related serine/threonine kinase gene Acatg1 significantly enhanced $\mathrm{CPC}$ yield through retaining PcbC and increasing the transcriptional levels of related genes [21]. Acatg1 is essential for the formation of autophagosome under starvation in A. chrysogenum. Acatg11 is involved in the selective autophagy pathway as a basic scaffold for phagosome assembly. However, deficiency of Acatg11 did not increase CPC production [22]. The relationship between autophagy and protein degradation should be complicated. How exactly autophagy participates in these fungal processes remains unknown.

In the present study, a S. cerevisiae ATG8 homologue gene Acatg8 was identified from A. chrysogenum. Gene disruption and genetic complementation revealed that Acatg8 is essential for autophagosome formation and autophagic process. Disruption of Acatg8 significantly reduced conidiation and fungal viability especially at the late stage of fermentation, but remarkably enhanced cephalosporin $\mathrm{C}$ yield. Through induced expression of Acatg8, the autophagic process was modulated and cephalosporin $C$ yield was remarkably increased in the case of maintaining fungal viability. This study provides a promising approach for increasing antibiotic yield through modulating autophagic process in A. chrysogenum.

\section{Materials and methods}

Strains, media, and growth conditions

Strains and plasmids used in this study were listed in Additional file 1: Table S1. For growth and conidiation of A. chrysogenum, TSA and LPE media were used respectively as described previously [22]. The modified MDFA medium was used for CPC production as described previously [23]. Czapek medium (per liter: $30.0 \mathrm{~g}$ sucrose, $3.0 \mathrm{~g} \mathrm{NaNO}_{3}, 0.5 \mathrm{~g} \mathrm{MgSO}_{4} \cdot 7 \mathrm{H}_{2} \mathrm{O}, 0.0125 \mathrm{~g} \mathrm{FeSO}_{4} \cdot 7 \mathrm{H}_{2} \mathrm{O}$, $0.5 \mathrm{~g} \mathrm{KCl}, 1.3 \mathrm{~g} \mathrm{~K}_{2} \mathrm{HPO}_{4} \cdot 3 \mathrm{H}_{2} \mathrm{O}, 15.0$ g agar), Czapek-N 
medium (Czapek medium without nitrogen source), Czapek-C medium (Czapek medium without carbon source) and WA medium (water with $1 \%$ agar) were used to detect the viability of $A$. chrysogenum and its derivatives. For Agrobacterium tumefaciens-mediated transformation (ATMT), minimal medium, co-cultivation medium and induction medium were used as described previously [23]. YPD medium (per litter: yeast extract $10.0 \mathrm{~g}$, tryptone $20.0 \mathrm{~g}$, glucose $20.0 \mathrm{~g}$, agar $20.0 \mathrm{~g}$ ) was used for the growth of S. cerevisiae BY4742 (the wild-type strain) and its derivatives. Nitrogen-starved medium (SG-N) (per litter: YNB $1.7 \mathrm{~g}$; Galactose 20.0 g; histidine $0.02 \mathrm{~g}$; leucine $0.1 \mathrm{~g}$; lysine $0.02 \mathrm{~g}$; uracil $0.02 \mathrm{~g}$ ) was used for detecting the viability of $S$. cerevisiae and its derivatives. Escherichia coli was used for propagating plasmids.

\section{RNA isolation, quantitative real-time PCR and western blotting}

Total RNA was isolated using Trizol Reagent (Invitrogen, USA) according to the commercial protocol and digested by DNase I to remove the genomic DNA as described previously [24, 25]. cDNA was obtained using the PrimeScript $^{\mathrm{TM}}$ RT Reagent Kit (TaKaRa). Synthesis cDNA and real-time RT-PCR were performed as described previously [24]. Western blot analysis of the isopenicillin $\mathrm{N}$ synthase PcbC was performed and the glyceraldehyde3-phosphate dehydrogenase (AcGapdh, GenBank accession No. MF383617) was used as control [21, 22].

\section{Identification of Acatg8 and heterologous complementation of the S. cerevisiae ATG8 mutant}

All primers used in this study were listed in Additional file 1: Table S2. We searched the genomic DNA sequence of A. chrysogenum CGMCC 3.3795 using the BLASTX program in the National Center for Biotechnology Information (NCBI). A query sequence, which encodes a putative protein showed $78 \%$ identity to Atg8 from S. cerevisiae, was designated Acatg8. To characterize Acatg8, the $A$. chrysogenum wild type strain (WT) was cultured in TSA liquid medium at $28^{\circ} \mathrm{C}$ on a rotary shaker $(220 \mathrm{rpm})$ for $48 \mathrm{~h}$. The supernatant was discarded after centrifugation at 12,000 rpm for $5 \mathrm{~min}$. After draining the mycelia with filter paper, liquid nitrogen was added to freeze the mycelia quickly. Then, the mycelia were crushed with sterilized pestle and mortar. DNA Quick Plant System (TianGen, China) and Trizol Reagent were used to isolate the fungal genomic DNA and total RNA respectively. DNA or cDNA of the speculated Acatg8 gene were amplified with primers Acatg8-F/Acatg8-R and inserted into the vector pEASY-Blunt (TransGen, Beijing) to generate $\mathrm{pEB}:: \mathrm{Acatg} 8$ and $\mathrm{pEB}:: \mathrm{CAcatg} 8$, respectively. The inserts of $\mathrm{pEB}::$ Acatg8 and $\mathrm{pEB}:: \mathrm{CAcatg} 8$ were verified by sequencing.
To complement the $S$. cerevisiae ATG8 mutant, the cDNA of Acatg8 was inserted into pYES2 (Invitrogen) under control of the yeast GAL1 promoter. The resulting plasmid pYES2::CAcatg8 was introduced into the S. cerevisiae ATG8 mutant using a small-scale yeast transformation protocol (Invitrogen, V825-20). Expression of Acatg8 in the transformants was verified by RT-PCR. Viability of the S. cerevisiae ATG8 mutant and its complemented strains was detected after incubation for 18 days on the nitrogen-starved medium (SG-N).

\section{Localization of AcAtg8 in A. chrysogenum}

The DNA fragment containing PAcatg8-GFP-Acatg8$\mathrm{T}$ was amplified from pCMVPEAT [21] with primers peatF/R. After digestion with $S w a \mathrm{I}$, the fragment was ligated into the corresponding sites of $\mathrm{pAgB}$ to give pAgB::PAcatg8-GFP-Acatg8-T. Finally, pAgB::PAcatg8GFP-Acatg8-T was introduced into WT through ATMT. An Axio-observer A1 microscope (Carl Zeiss) was used for microscope observation. Zeiss AxioCam MR camera was used to capture the images of cells. For editing images, AxioVision software and Adobe-Photoshop CS3 software were used.

\section{Constructions of the Acatg8 disruption mutant and its complemented strain}

To construct the Acatg8 disruption mutant, a 5480 bp DNA fragment containing Acatg8 was amplified from WT with primers Acatg8DF/Acatg8DR and inserted into pEASYBlunt (TransGen, Beijing) to generate pEB::Acatg8LR. After verified by sequencing, pEB::Acatg8LR was digested by Sall. The $1.5 \mathrm{~kb}$ Sall DNA fragment containing the bleomycin resistant gene (ble) from pJLRNAi was inserted into the corresponding site of $\mathrm{pEB}:$ :Acatg8LR to give the plasmid pEB::Acatg8LR-B. Then, pEB::Acatg8LR-B was digested by $S w a \mathrm{I}$ and the DNA fragment containing Acatg8-ble was inserted into pAg1H3. The resulting plasmid pAg::Acatg8LR-B was introduced into WT via ATMT as described previously [22]. After 3 days of co-incubation at $24{ }^{\circ} \mathrm{C}$, the bleomycin resistant and hygromycin $\mathrm{B}$ sensitive transformants were selected as the Acatg8 disruption mutant ( $\triangle$ Acatg8). Finally, $\triangle$ Acatg8 was verified by PCR with gene outside primers Acatg8-outF/R and Southern hybridization.

For genetic complementation, a 2331 bp DNA fragment containing the complete Acatg 8 was amplified by PCR with primers Acatg8C-F/Acatg8C-R and subcloned into the SwaI site of pAg1H3 to give pAg::Acatg8C. Finally, pAg::Acatg8C was introduced into $\triangle$ Acatg8 via ATMT and the transformants were selected in the TSA medium with $50 \mu \mathrm{g} \mathrm{ml}^{-1}$ hygromycin $\mathrm{B}$. The transformants were further verified by RT-PCR. One of them was 
randomly selected as the complemented strain (Acatg8C) and used in subsequent experiments.

\section{Inducible expression of AcAtg8 in $\Delta$ Acatg8}

For inducible expression of Acatg8 in $\triangle$ Acatg8, a $1.5 \mathrm{~kb}$ DNA fragment containing the xylose/xylan-inducible promoter $\left(x_{1} l^{\mathrm{P}}\right)$ was amplified with primers $x y{ }^{\mathrm{P}}-\mathrm{F} / \mathrm{R}$ and inserted into the HindIII site of pEASY-Blunt, to give the plasmid $\mathrm{pEB}:: \mathrm{xyl}^{\mathrm{P}}$. The Acatg8 terminator region was amplified with primers Ter-F/R and inserted into the BamHI site of pEASY-Blunt, to give the plasmid pEB::T. The green fluorescent protein (GFP) encoding gene without stop codon was amplified from the plasmid pEGFP$\mathrm{N} 1$ (Clontech) with primers gfpF/gfpNR and inserted into the BglII site of pEASY-Blunt to give the plasmid pEB::GFP. The Acatg8 without stop codon was amplified from the cDNA of $A$. chrysogenum with primers atg8F/R and inserted into the $X b a \mathrm{I}$ site of pEASY-Blunt to give the plasmid pEB::CAcatg8. The $\mathrm{xyl}^{\mathrm{P}}{ }^{\mathrm{p}}, \mathrm{GFP}$, Acatg8 and the terminator region were in turn ligated into $\mathrm{PCM}-$ V3xFlag-10 to generate pCMV::xyl ${ }^{\mathrm{P}}$-GFP-Acatg8-T. After digested with SwaI, the DNA fragment containing $\mathrm{xyl}^{\mathrm{P}}$-GFP-Acatg8-T was ligated into the corresponding sites of pAg1H3. Finally, the resulting plasmid pAg::xyl ${ }^{\mathrm{P}}$ GFP-Acatg8- $T$ was introduced into $\Delta$ Acatg8 through ATMT. For inducible expression of Acatg8, the resulting strain $\triangle$ Acatg8/pAg::xyl ${ }^{\mathrm{P}}$-GFP-Acatg8-T was incubated in the LPE or modified MDFA medium supplemented with $1 \%$ xylose.

\section{Detection of autophagy}

Transmission electron microscopy (TEM) was used for detecting the fungal autophagy as described previously $[21,26]$. For monodansyl cadavarine (MDC) dye analysis, $1 \times 10^{6}$ conidia of WT, $\triangle$ Acatg8 and Acatg8C were added to $20 \mathrm{ml}$ of TSA medium and incubated at $28{ }^{\circ} \mathrm{C}$ for $20 \mathrm{~h}$. The mycelia were harvested and washed with sterilized distilled water for three times, and then transferred into distilled water supplemented with $2 \mathrm{mM}$ of phenylmethylsulfonyl fluoride (PMSF). The fungal cultures were collected after $4 \mathrm{~h}$ incubation and stained with MDC (Sigma-Aldrich, D-4008) at a final concentration of $60 \mu \mathrm{M}$ for $30 \mathrm{~min}$ in the dark. After rinsed three times with water, samples were observed under fluorescence microscopy.

\section{Detection of fungal conidiation and cephalosporin $C$ production}

Conidiation was detected as described previously [27]. Fermentation of $A$. chrysogenum and detection of CPC production were performed as described previously [21, 28]. Bacillus subtilis CGMCC 1.1630 was used as the indicator strain of CPC production.

\section{Detection of PcbC and CefD2}

For detecting expression of $p c b C$, a plasmid containing the PcbC-GFP fusion protein encoding gene was constructed. The $p c b C$ coding region and its promoter region were amplified by PCR with primers Ppcbc-F/R and $\mathrm{pcbC}-\mathrm{F} / \mathrm{R}$, respectively. After verified by sequencing, the amplified fragments were introduced into pCMVGFP-T to give pCMV::PpcbC-pcbC-GFP-T. For detecting expression of $c e f D 2$, the same strategy was carried out. The cefD 2 coding region and its promoter region were amplified with primers PcefD2-F/R and cefD2-F/R, respectively. After verified by sequencing, the amplified fragments were introduced into pCMV-GFP-T to give pCMV::PcefD2-GFP-cefD2-T. Then, the fragments containing PpcbC-pcbC-GFP-T and PcefD2-GFP-cefD2-T were amplified from $p C M V:: P p c b C-p c b C-G F P-T$ and pCMV::PcefD2-GFP-cefD2-T respectively and inserted into $\mathrm{pAgHB}$ to generate $\mathrm{pAg}: \mathrm{PpcbC}$-pcbC-GFP-T and pAg::PcefD2-GFP-cefD2-T. Finally, these two plasmids were introduced into WT and $\Delta$ Acatg8, respectively. The expressions and localizations of PcbC and CefD2 were observed under fluorescence microscopy.

\section{Detection of pexophagy and mitophagy}

Detection of pexophagy and mitophagy in A. chrysogenum was performed as described previously [22].

\section{Results}

Identification of the ATG8 homologue Acatg8 from $A$. chrysogenum

Since Atg8 proteins are highly conserved in fungi [29], an open reading frame (ORF) encoding an Atg8 homologous protein was identified in $A$. chrysogenum and it was designated Acatg8 (GenBank accession No. KJ569771). The cDNA of Acatg8 was amplified and sequenced. Comparing the sequences of Acatg8 and its cDNA, two introns (localized in positions +93 to +284 and +504 to +566 with respect to the translation initiation site) were found (Fig. 1a). The deduced protein of Acatg 8 contains 118 amino acids, and its theoretical molecular weight is $13.7 \mathrm{kDa}$. Based on sequence alignment, AcAtg8 shows 96\% identity to Atg8 from A. oryzae, 83\% identity to Atg8 from Ustilago maydis, 69\% identity to Atg8 from Dictyostelium discoideum, 91\% identity to Atg8 from M. oryzae, 78\% identity to Atg8 from S. cerevisiae (Fig. 1b).

To address whether AcAtg8 has the same physiological function in autophagic process as Atg8 of S. cerevisiae, the plasmid pYES2::Acatg8 was constructed and introduced into the $S$. cerevisiae ATG8 mutant ( $\Delta$ atg8) which generally dies after a long time incubation under starvation condition. Three heterologous complemented strains of $\Delta \mathrm{atg} 8$, designated as $\mathrm{YC} 1-3$, were obtained through uracil selection and Acatg 8 expression was confirmed by 
a

\section{ATG}

TGA
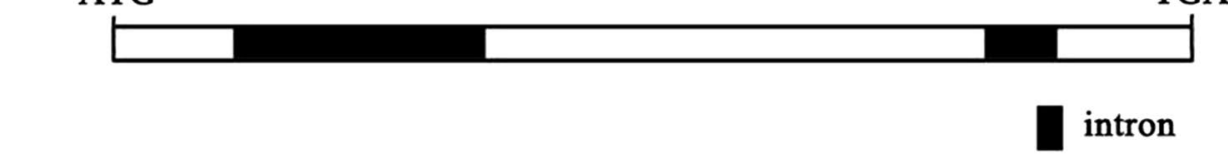

b

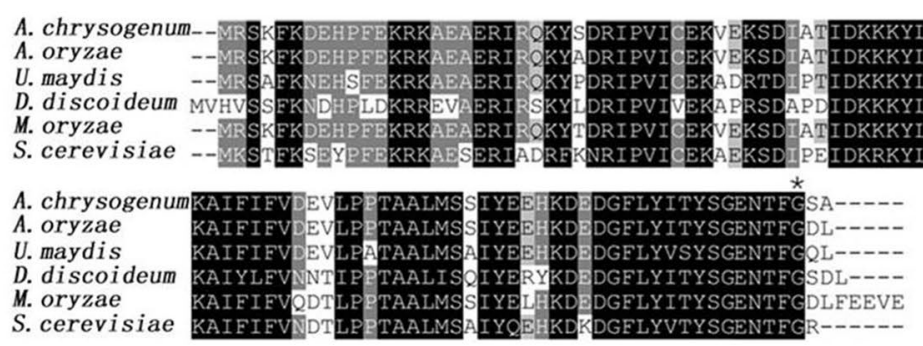

C

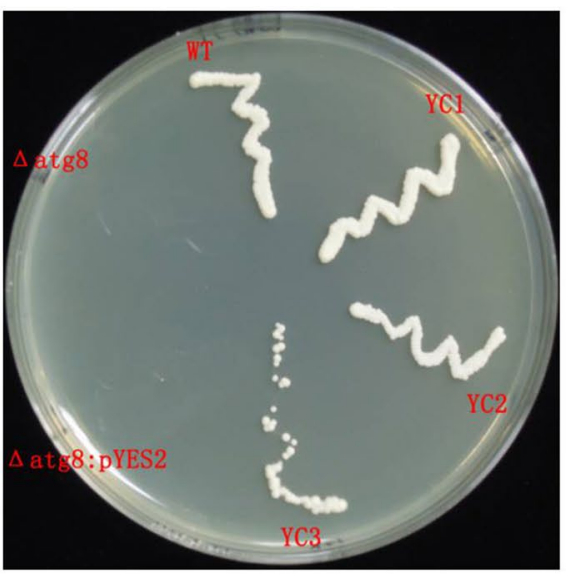

d
DIC
GFP-Acatg8

Nonstarved

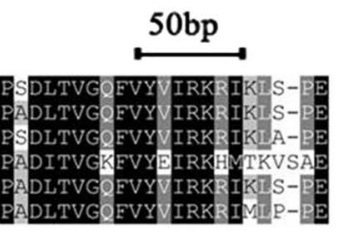

Merge
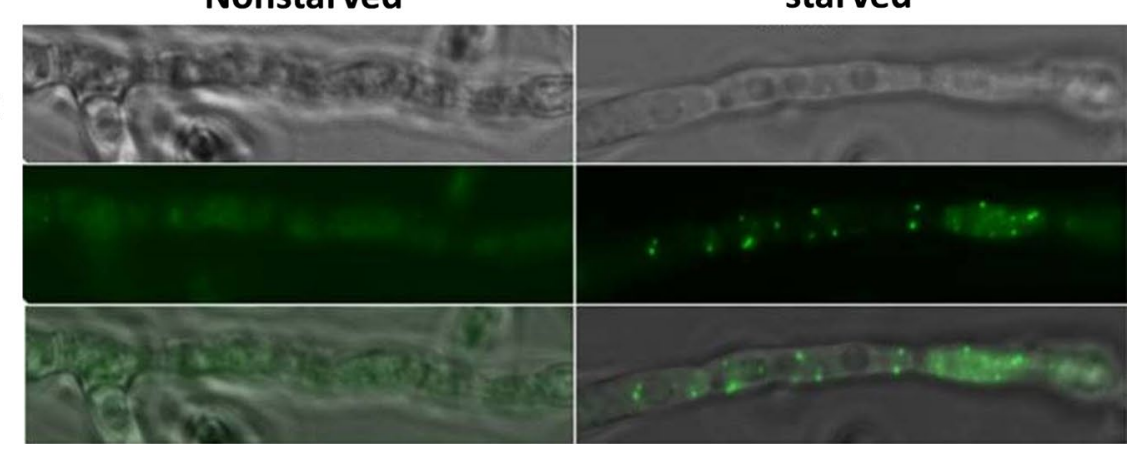

Fig. 1 Identification of an ATG8 gene homologue from A. chrysogenum. a Acatg 8 with 2 introns. b Sequence alignment of AcAtg8 with its homologs. AcAtg8 shows 96\% identity to Atg8 from A. oryzae, $83 \%$ identity to Atg8 from U. maydis, 69\% identity to Atg8 from D. discoideum, $91 \%$ identity to Atg 8 from M. oryzae, $78 \%$ identity to Atg 8 from S. cerevisiae. The asterix indicates the glycine cutting site that is conserved at the $C$ terminal. c Viability of $\triangle \mathrm{atg} 8$ and its complemented strains. The viability of $\Delta$ atg 8 and its complemented strains was detected after 18 days of incubation on the nitrogen-starved medium (SG-N). $\mathbf{d}$ Distribution of AcAtg8 in A. chrysogenum. Fluorescence observation demonstrated that AcAtg8 was widely distributed throughout the hyphae when the fungal strains grew under nutrient-rich conditions (Nonstarvation), while AcAtg8 was transferred into vacuoles under starvation condition (starvation). WT: the S. cerevisiae wild-type strain; $\triangle$ atg8: the S. cerevisiae ATG8 mutant; YC1-3: the complemented strains of $\triangle \mathrm{atg} 8$; $\triangle \mathrm{atg} 8 / \mathrm{pYES2}$ : $\triangle$ atg8 carrying the plasmid pYES2 as the control; DIC; differential interference contrast; GFP: green fluorescent protein 
RT-PCR (Additional file 1: Fig. S1). After 18 days of incubation on the nitrogen-starved medium (SG-N) at $30{ }^{\circ} \mathrm{C}$, the $S$. cerevisiae wild-type strain (WT), $\Delta$ atg8 and $\mathrm{YC} 1-3$ were shifted to YPD medium. Both WT and YC1-3 grew well, but $\Delta$ atg 8 and $\Delta$ atg $8 /$ pYES2 could not grow due to a long time nitrogen starvation (Fig. 1c), indicating that AcAtg8 complements the atg8 mutation in S. cerevisiae. Combined with the sequence alignment analysis, Acatg8 is the homologous gene of S. cerevisiae ATG8.

\section{Acatg8 is essential for the autophagic process of $A$. chrysogenum}

To detect the distribution of AcAtg8 in A. chrysogenum, pAgB::GFP-AcAtg8 used for GFP-Acatg8 expression was constructed and introduced into WT. The verified transformant was incubated in TSA medium for $16 \mathrm{~h}$ and then shifted into the sterile water containing $2 \mathrm{mM}$ of phenylmethylsulfonyl fluoride (PMSF) for additional $4 \mathrm{~h}$ incubation. AcAtg8 was widely distributed throughout the hyphae under nutrient-rich growth conditions (Nonstarvation), while it was punctually localized under starvation growth conditions (starvation) (Fig. 1d). The distribution of AcAtg8 in A. chrysogenum resembles that of Atg8 in S. cerevisiae [30].

To further address its function, Acatg8 was disrupted in $A$. chrysogenum via homologous recombination (Additional file 1: Fig. S2). The Acatg8 disruption mutant ( $\triangle$ Acatg8) was verified by PCR and Southern hybridization, respectively (Additional file 1: Fig. S2). After transferred into the starvation condition, WT, $\Delta$ Acatg8 and the complemented strain (Acatg8C) were cultured for 4 h. Transmission electron microscopy (TEM) demonstrated that autophagosomes were localized in the vacuoles of WT but not in $\triangle$ Acatg8, indicating that disruption of Acatg8 inhibits autophagic process of $A$. chrysogenum (Fig. 2a). In consistent with TEM observation, monodansyl cadavarine (MDC) analysis demonstrated that autophagosomes were localized in the vacuoles of WT, but not in the vacuoles of $\Delta$ Acatg8 (Fig. 2b). Acatg8C restored the wild-type phenomenon.

\section{Growth, conidial germination and conidiation were reduced in $\triangle$ Acatg 8}

Since $A T G 8$ is essential for yeast survival under starvation condition, the effect of Acatg 8 deficiency on the growth of $A$. chrysogenum was detected under nutrientstarvation condition. After grown on TSA medium for 3 days, the hyphae of WT, $\triangle$ Acatg8 and Acatg8C were collected and transferred into Czapek medium, Czapek-N medium (No nitrogen), Czapek-C medium (No carbon) and WA medium (water with agar 1\%) respectively and incubated for additional 7 days. Comparing the colonial diameters of different strains demonstrated

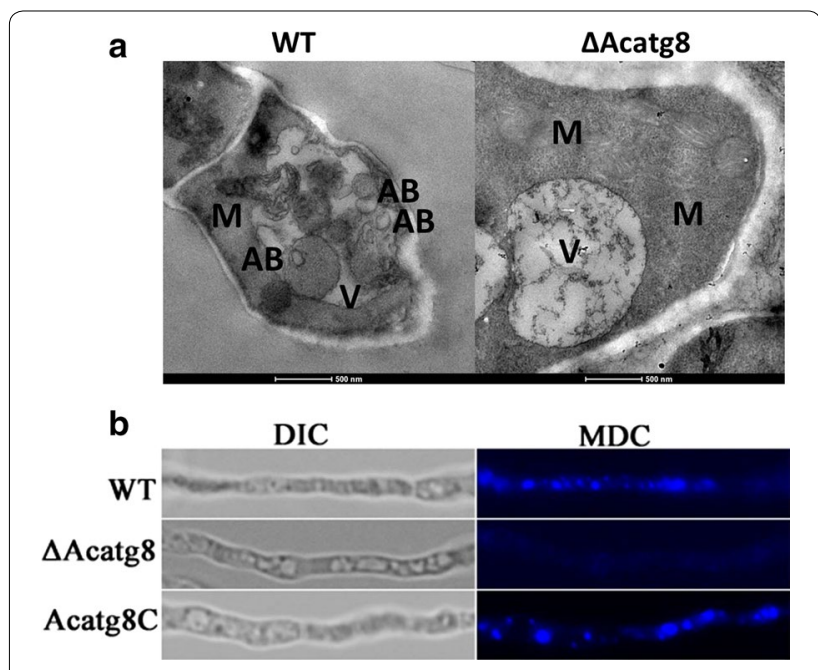

Fig. 2 Effect of Acatg8 deficiency on autophagy of A. chrysogenum. a Transmission electron microscopy (TEM) analysis of WT and $\triangle$ Acatg 8 under nutrition-shift condition. The autophagosomes were observed in the vacuole of WT, but not in that of $\triangle$ Acatg8 under starvation condition. V: vacuole; AB: autophagic body; M: mitochondria. Bars, $500 \mathrm{~nm}$. b Distribution of autophagosomes in WT, $\triangle$ Acatg 8 and Acatg8C. The autophagosomes were observed in the vacuoles of WT, but not in the vacuoles of $\triangle$ Acatg8. The complemented strain restored the wild-type phenomenon. Images were observed after $4 \mathrm{~h}$ induction. DIC: differential interference contrast; MDC: monodansyl cadavarine

that $\triangle$ Acatg8 grew slower than $\mathrm{WT}$ and Acatg8C, and $\Delta$ Acatg8 hardly grew on WA medium (Fig. 3a). These results indicated that Acatg8 is important for the growth of $A$. chrysogenum under starvation condition.

When grown on LPE medium, spore germination of $\triangle$ Acatg8 was delayed compared with that of WT and Acatg8C (Fig. 3b). It is possible that autophagy is also involved in spore germination of $A$. chrysogenum. The plasmid pAg::PAcatg8-GFP-Acatg8-T used for GFPAcatg8 expression was constructed and introduced into WT, and germination of the verified transformant was detected under fluorescence microscope. Punctates of GFP-AcAtg8 were observed during spore germination, implying autophagosomes were formed (Additional file 1: Fig. S3). It is speculated that fungal cells degrade the stored nutrients (glycogen, fat droplets, etc.) through the autophagic process when the spores rapidly germinate under appropriate conditions. Similar with that found in $M$. oryzae [31], disruption of Acatg8 dramatically reduced conidiation of $A$. chrysogenum (Fig. $3 \mathrm{c}$ and 3d). Meanwhile, the transcript levels of $A c b r l A, A c w e t A$ and $A c a b a A$, as the key genes for conidiation of $A$. chrysogenum [27], were evaluated. A non-conidiation related gene glyceraldehyde-3-phosphate dehydrogenase encoding gene AcGapdh (GenBank accession No. MF383617) of A. chrysogenum was used as control. Transcriptional 

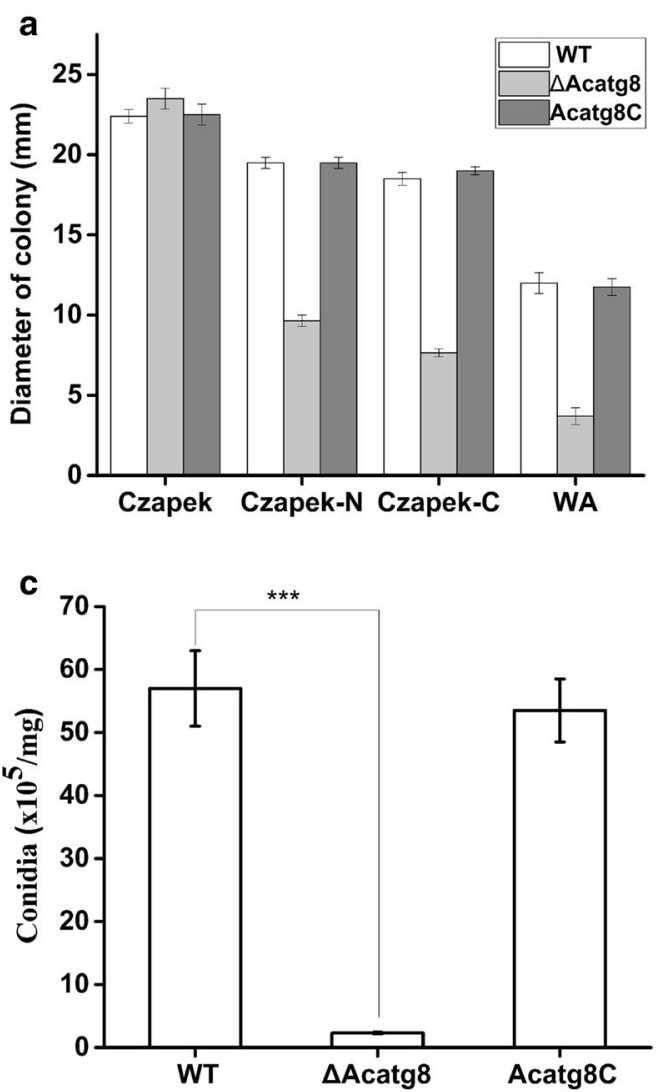

b

$7 \mathrm{~h}$

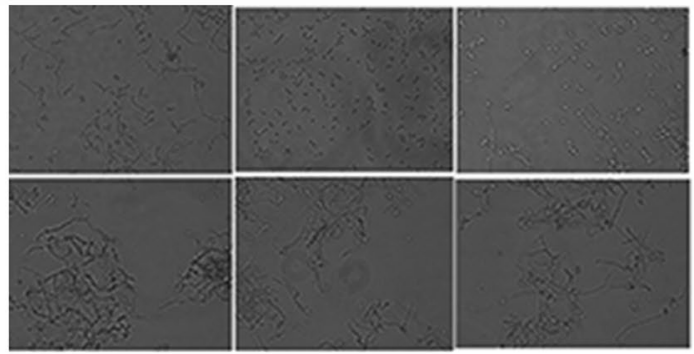

WT

$\Delta$ Acatg8

Acatg8C

d

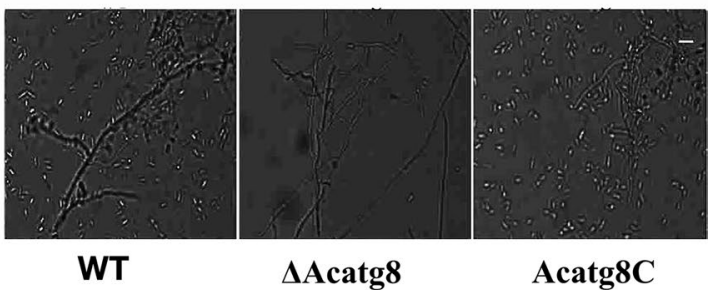

Fig. 3 Growth, conidial germination and conidiation of WT, $\triangle$ Acatg 8 and Acatg $8 C$. a Growth of WT, $\triangle A c a t g 8$ and Acatg8C was detected. After incubated in TSA medium for 72 h, hyphae of WT, $\triangle A$ catg 8 and Acatg 8 C were transferred to Czapek medium, Czapek-N medium (No nitrogen), Czapek-C medium (No carbon) and WA medium (water with agar 1\%). The colony diameters of different strains were measured. The asterix indicates that the differences between strains are significant. $p<0.001^{* *} ; p<0.01^{* *} ; p<0.05^{*}$. b Disruption of Acatg8 reduces conidial germination. When grown on LPE medium, the conidial germination of $\triangle$ Acatg8 was delayed compared with that of WT and Acatg8C. $\mathbf{c}$ Disruption of Acatg8 reduces fungal conidiation. The number of conidia in $\triangle$ Acatg 8 grown on LPE medium for 7 days was only about $5 \%$ of that in WT. Error bars show standard deviations of three independent experiments. The asterix indicates that the differences between strains are significant. $p<0.001^{* * *}$; $\mathrm{p}<0.01^{* *} ; \mathrm{p}<0.05^{*}$. d Conidiation of WT, $\triangle$ Acatg 8 and Acatg8C on LPE medium for 7 days was observed under microscope

analysis revealed that disruption of Acatg8 significantly decreased the transcriptional level of AcbrlA,AcwetA and AcabaA (Additional file 1: Fig. S4). These results indicated that Acatg8 is important not only for the growth and conidial germination, but also for the fungal conidiation of A. chrysogenum.

\section{Addition of exogenous carbon sources partially restores the conidiation of $\Delta$ Acatg8}

Combined with the results above, we speculated that $\Delta$ Acatg8 could not recycle its own cellular components due to autophagic defect. Therefore, $\Delta$ Acatg8 could not provide enough nutrients or energy for conidiation. To verify our speculation, exogenous nitrogen and carbon sources were added in LPE medium. As expected, supplementation of exogenous carbon sources partially restored the conidiation of $\Delta$ Acatg8 (Table 1). However, supplementation of exogenous nitrogen sources could not restore the conidiation of $\Delta$ Acatg8 (Table 1). It has been reported that the conidial formation was affected by the cellular glycogen in M. oryzae [31]. In M. oryzae, disruption of MoATG8 dramatically reduced the conidial formation and exogenous supply of glucose or deletion of a glycogen phosphorylase Gph1 could suppress the conidiation defects, indicating glycogen homeostasis is important for fungal conidiation. Like in M. oryzae, carbon source such as glucose in the culture medium may affect glycogen homeostasis or the enzymes involved in glycogen metabolism, and in turn partially recover the phenotype caused by autophagic defect in $\Delta$ Acatg8.

\section{Cephalosporin C production is significantly increased in $\Delta$ Acatg8}

Our previous study showed that disruption of Acatg1 increased CPC yield through reducing degradation of cephalosporin biosynthetic proteins [21], indicating 
Table 1 Effects of supplemented carbon/nitrogen sources on conidiation of $A$. chrysogenum

\begin{tabular}{|c|c|c|c|}
\hline \multirow{2}{*}{$\begin{array}{l}\text { Supplemented } \\
\text { sources }\end{array}$} & \multicolumn{3}{|c|}{ Conidia ( $\times 10^{7} /$ plate) } \\
\hline & $\overline{W T}$ & $\Delta$ Acatg8 & Acatg $8 C$ \\
\hline \multicolumn{4}{|l|}{ Carbon sources } \\
\hline CK & 13.92 & $0.98^{* *}$ & 13.85 \\
\hline Glucose & 13.49 & $7.75^{*}$ & 12.51 \\
\hline Sucrose & 12.06 & $7.62^{*}$ & 12.96 \\
\hline Inositol & 11.75 & $5.86^{*}$ & 10.95 \\
\hline Mannitol & 10.65 & $6.86^{*}$ & 11.92 \\
\hline \multicolumn{4}{|l|}{ Nitrogen sources } \\
\hline CK & 13.92 & $0.98^{* *}$ & 13.85 \\
\hline $\mathrm{NH}_{4} \mathrm{Cl}$ & 5.96 & $0.89^{* *}$ & 5.26 \\
\hline $\mathrm{NaNO}_{3}$ & 4.75 & $0.85^{* *}$ & 4.47 \\
\hline $\mathrm{NH}_{4} \mathrm{NO}_{2}$ & $2.82^{*}$ & $1.07^{* *}$ & 3.73 \\
\hline Met & $2.94^{*}$ & $1.97^{*}$ & 2.88 \\
\hline Pro & 3.84 & $1.63^{* *}$ & 2.76 \\
\hline
\end{tabular}

Conidia formation in WT, $\triangle$ Acatg8 and Acatg 8 C grown on the LPE medium supplemented with different carbon/nitrogen sources. Glucose, sucrose, inositol or mannitol was supplemented at the final concentration of $1 \%$. Ammonium chloride $\left(\mathrm{NH}_{4} \mathrm{Cl}\right)$, niter nitrate $\left(\mathrm{NaNO}_{3}\right)$, ammonium nitrate $\left(\mathrm{NH}_{4} \mathrm{NO}_{2}\right)$, methionine (Met) or proline (Pro) was added in the LPE medium at the final concentration of $1 \%$. Numbers of conidia were counted after 7 days incubation. CK, the medium without supplementation of carbon/nitrogen sources. The data are derived from the average of three independent experiments. The asterix indicates that the differences between strains are significant. $p<0.001^{* * *} ; p<0.01{ }^{* *} ; p<0.05^{*}$

deficiency of autophagy facilitates antibiotic production. Therefore, the CPC production of $\triangle A$ catg8 was detected. As expected, the CPC production increased threefold in $\triangle$ Acatg8 compared with that in WT (Fig. 4a). What excites us most is that not only the yield of CPC was increased, but also CPC was produced earlier in $\Delta$ Acatg8. Only $12 \mathrm{~h}$ fermentation, $\Delta$ Acatg8 started to produce appreciable quantity of CPC in MDFA medium. While the CPC production was hardly detected in WT before $48 \mathrm{~h}$ fermentation. Unfortunately, disruption of Acatg8 significantly decreased the fungal biomass (Fig. 4b). Furthermore, transcript levels of the key genes $(p c b A B, p c b C, c e f D 1$, cefD2, cefEF and $c e f G$ ) for cephalosporin biosynthesis in $A$. chrysogenum were measured by real time RT-PCR (Fig. 5). In consistence with $\mathrm{CPC}$ production, the transcription of these key genes was maintained continuously at a relatively high level in $\Delta$ Acatg8 from 12 to $48 \mathrm{~h}$. These results indicated that at least one of the reasons for the yield increase and earlier production of CPC was due to the elevated expression of cephalosporin biosynthetic genes. However, the transcription of these key genes in $\Delta$ Acatg8 was quickly declined after $96 \mathrm{~h}$ fermentation and the transcription level was even lower than that in WT. To explain the contradiction between CPC yield and the biosynthetic gene transcripts at the late stage of fermentation, the degradation of $\mathrm{PcbC}$ was detected in $\triangle$ Acatg8 and WT.

Western blot analysis revealed that the quantity of PcbC in $\triangle$ Acatg8 was higher than that in WT not only at the early stage but also at the late stage of fermentation (Fig. 6a), suggesting accumulation of $\mathrm{PcbC}$ is one of the reasons for cephalosporin yield increment in $\Delta$ Acatg8. Fluorescence observation further demonstrated that there was no fluorescence in the vacuoles of WT and $\Delta$ Acatg8 at the early stage of fermentation. At the late stage of fermentation, the vacuoles of WT were filled with fluorescence, indicating most of the PcbC was transferred into vacuoles for degradation. While there was no fluorescence in the vacuoles of $\Delta$ Acatg8 and PcbC was accumulated in the cytoplasm (Fig. 6b), indicating PcbC was retained in $\triangle$ Acatg8. Therefore, it is possible that deficiency of autophagy reduces the degradation of cephalosporin biosynthetic proteins and in turn increases $\mathrm{CPC}$ production at the late stage of fermentation in A. chrysogenum.

\section{Disruption of Acatg8 dramatically reduces fungal viability at the late stage of fermentation}

It is interesting to find that disruption of Acatg 8 remarkably increased CPC production. However, the fungal biomass demonstrated the viability of $\Delta$ Acatg8 was significantly reduced especially at the late stage of fermentation (Fig. 4b). These results further indicated that Acatg8 is very important for fungal survival and related with CPC production. It is reasonable that WT can degrade cellular components and recycle nutrient through autophagy at the late stage of fermentation, while $\Delta$ Acatg8 could not since its autophagic process is inhibited. In addition, many toxic substances especially reactive oxygen species (ROS) were gradually accumulated along with the metabolic process during extension of cell survival time. The accumulation of ROS is speculated as an important factor for causing the death of $\Delta$ Acatg8. To confirm our speculation, the citric acid dehydrogenase (Cit) fused with GFP was used to mark mitochondria (Additional file 1: Fig. S5). The fluorescence observation showed that mitochondria of WT were almost degraded in vacuoles at the late stage of fermentation, while no fluorescence was observed in vacuoles of $\Delta$ Acatg8 and a large number of mitochondria were accumulated in the cytoplasm of $\Delta$ Acatg8. Thus, disruption of Acatg 8 not only caused autophagic defect but also blocked the normal degradation of mitochondria. The accumulation of mitochondria, especially those dysfunctional mitochondria, could lead to the accumulation of ROS which causes premature death of $\Delta$ Acatg 8 . 


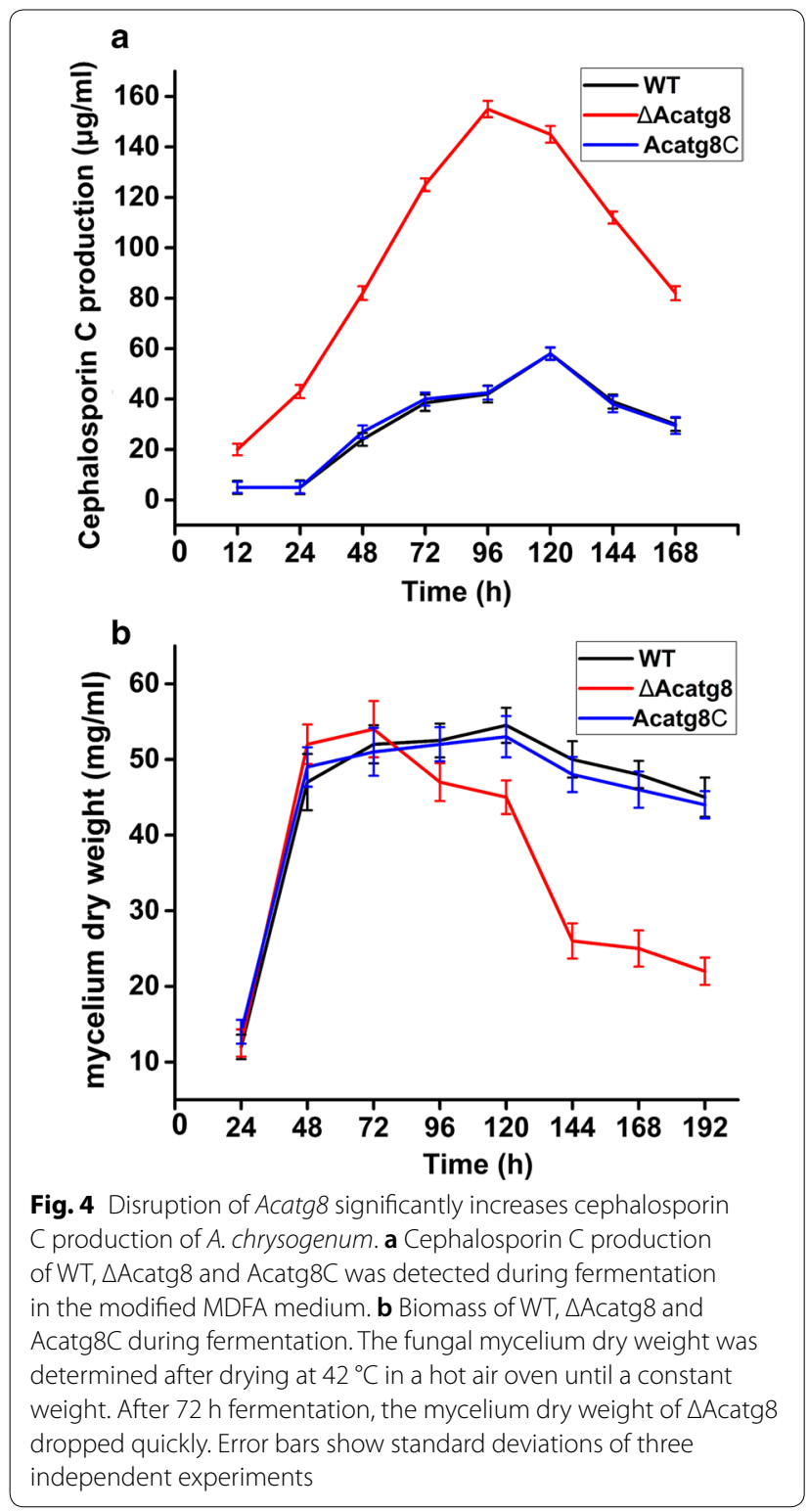

Inducible expression of Acatg8 in $\Delta$ Acatg 8 improves fungal viability but maintains high cephalosporin $C$ yield at the late stage of fermentation

The premature death is unfavorable for CPC production, especially in industry. To overcome the premature death of $\Delta$ Acatg8, the endogenous xylose/ xylan-inducible promoter $\mathrm{xyl}^{\mathrm{P}}$ was used for inducible expression of Acatg8. The promoter $\mathrm{xyl}^{\mathrm{P}}$, which is induced by xylose/xylan but repressed by glucose, has been successfully used in A. chrysogenum [32]. The plasmid pAg::xyl ${ }^{\mathrm{P}}$-GFP-Acatg8-T was constructed and introduced into $\triangle$ Acatg8 (Additional file 1: Fig. S6). As expected, $\Delta$ Acatg8/pAg::xyl ${ }^{\mathrm{P}}$-GFP-Acatg8-T dramatically increased fungal conidial formation in LPE plates supplemented with $1 \%$ xylose. The number of conidia in $\Delta$ Acatg8/pAg::xyl ${ }^{\mathrm{P}}$-GFP-Acatg8-T was about the same as that in WT (Additional file 1: Fig. S6). Then the expression of GFP in $\triangle \mathrm{Acatg} 8 / \mathrm{pAg}: \mathrm{xyl}^{\mathrm{P}}$-GFP-Acatg8$\mathrm{T}$ was detected at the sixth day of fermentation. Fluorescence observation indicated that GFP was expressed under control of $\mathrm{xyl}^{\mathrm{P}}$ at the late stage of fermentation when glucose in the medium was depleted (Additional file 1: Fig. S7). In consistent with the expression of GFP, Acatg8 was expressed in the presence of xylose when the non-inducing carbon source glucose was depleted in $\triangle$ Acatg8/pAg::xyl ${ }^{\mathrm{P}}$-GFP-Acatg8-T.

As mentioned above, the biomass of $\Delta$ Acatg 8 was only about $60 \%$ of WT at the late stage of fermentation due to the autophagic defect. When Acatg8 was expressed in $\Delta$ Acatg8/pAg::xyl ${ }^{\mathrm{P}}$-GFP-Acatg8-T, the fungal viability was restored to the wild-type level just like the conidial formation (Fig. 7a). The CPC production of $\triangle$ Acatg8/ pAg::xyl ${ }^{\mathrm{P}}$-GFP-Acatg8-T was also detected. Like $\triangle$ Acatg8, $\triangle$ Acatg8/pAg::xyl ${ }^{\mathrm{P}}$-GFP-Acatg8-T still kept the ability to produce more $\mathrm{CPC}$ in presence of xylose (Fig. 7b). Although it was lower than that of $\triangle$ Acatg8 at $96 \mathrm{~h}$ fermentation, the CPC yield of $\triangle$ Acatg8/pAg::xyl ${ }^{\mathrm{P}}$ GFP-Acatg8-T maintained at the high level during prolonged fermentation.

\section{Discussion}

Autophagy is the highly conserved eukaryotic physiological process which plays a vital role in maintaining intracellular carbon and nitrogen homeostasis [33]. Previous study showed that autophagy was related with morphological differentiation and antibiotic production in filamentous fungi $[15,21]$. During fermentation, the morphological differentiation of the high CPC producing strain proceeded rapidly. Swollen hyphal fragments and arthrospores appeared at $72 \mathrm{~h}$ fermentation [34]. In contrast with the arthrospore formation, an inverse relation was found between the fungal growth and $\mathrm{CPC}$ production [35]. Combining our results that inhibition of autophagy increased CPC production but decreased the fungal viability, it is possible that the autophagy was reduced in the high $\mathrm{CPC}$ producing strain.

In this study, the physiological function of core autophagy-related gene Acatg8 was investigated. Disruption of Acatg8 resulted in a significant increment of CPC yield. However, the fungal viability and conidiation were remarkably reduced in $\Delta$ Acatg8 due to the autophagic defect. To overcome the defects of the fungal viability and conidiation, the xylose/xylan-inducible promoter $\mathrm{xyl}^{\mathrm{P}}$ was used to control the expression of Acatg8 in $\Delta$ Acatg8. Through inducible expression of Acatg8 in $\Delta$ Acatg8, the fungal conidiation and growth were restored to the 


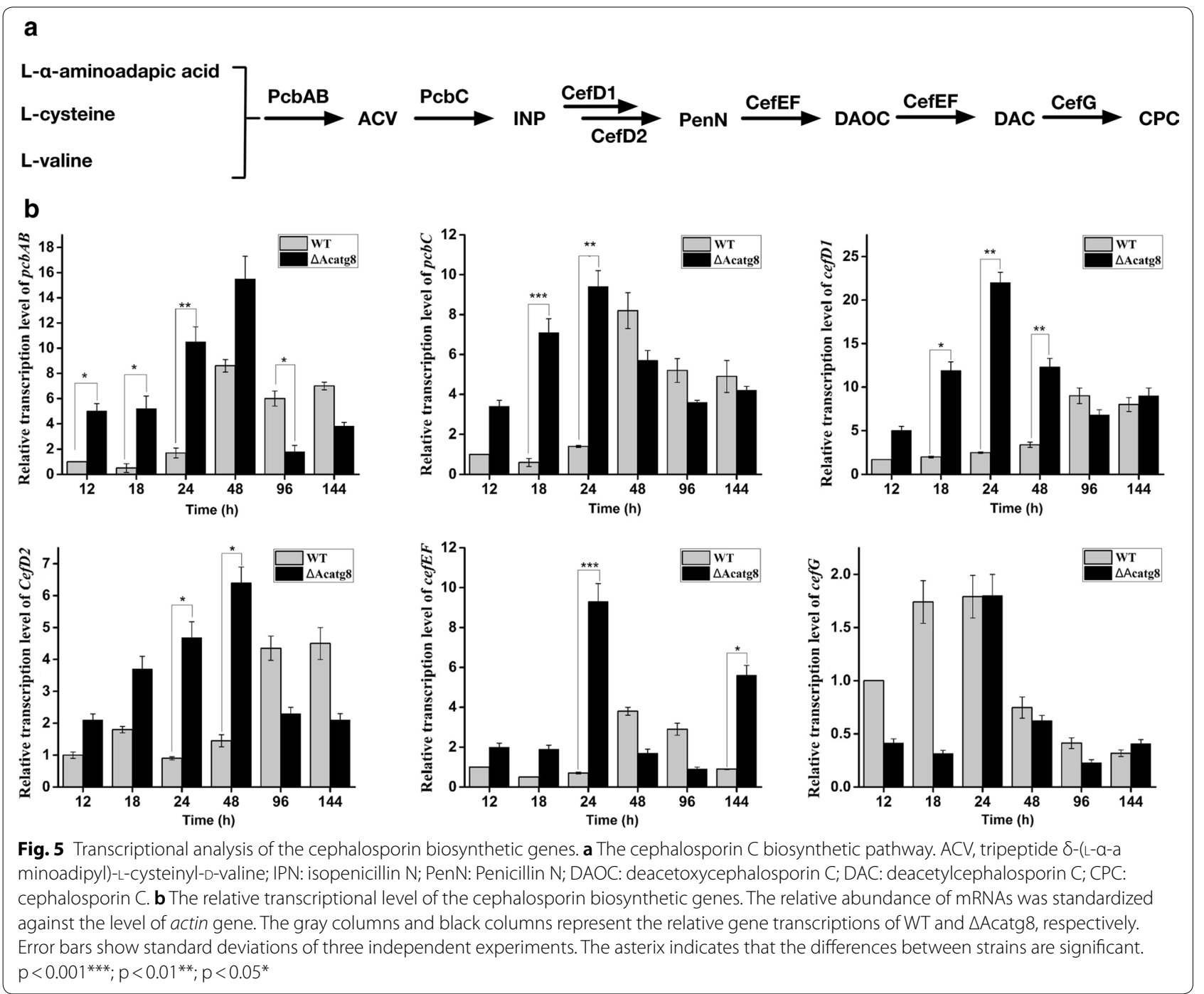

wild-type level, while the CPC production still maintained at a high level as we expected.

Peroxisomes are single-membrane organelles not only involved in the $\beta$-oxidation of fatty acids but also involved in secondary metabolite biosynthesis [36]. In $P$. chrysogenum, the final steps for conversion of isopenicillin $\mathrm{N}$ to penicillin $\mathrm{G}$ occur in peroxisomes $[37,38]$. It was also found that the high penicillin producing strains contain more peroxisomes and increasing peroxisome numbers by overexpression of pex 11 increased penicillin production 2-3 fold $[39,40]$. Sequence analysis revealed that some essential proteins for cephalosporin biosynthesis contain putative peroxisomal targeting signals (PTS), indicating that peroxisomes are also involved in CPC production [41]. It is reasonable that not only enhancing peroxisome proliferation but also inhibiting peroxisome degradation could increase peroxisome numbers. Disruption of Acatg8 clearly inhibited peroxisome degradation and increased peroxisome accumulation (Additional file 1: Fig. S8). Since CefD2 is localized in peroxisomes, increase peroxisome numbers could enhance the concentration of CefD2. As expected, fluorescence observation demonstrated CefD2 retained in $\triangle$ Acatg8 even after $144 \mathrm{~h}$ fermentation (Additional file 1: Fig. S9). We speculate that peroxisome accumulation is one of the main reasons for the CPC enhancement in $\triangle \mathrm{Acatg} 8$. However, the peroxisome accumulation through disruption of Acatg11 did not increase CPC production [22]. It is possible that Acatg11 has multiple functions and some of them are positive related with CPC production.

In S. cerevisiae, autophagy is the main mechanism for maintaining cellular survival under starvation condition $[42,43]$. Fungal cells tend to die once autophagy is inhibited $[44,45]$. In filamentous fungi, autophagy is used for recycling the carbon and nitrogen sources and reconstituting the intracellular components during fungal 


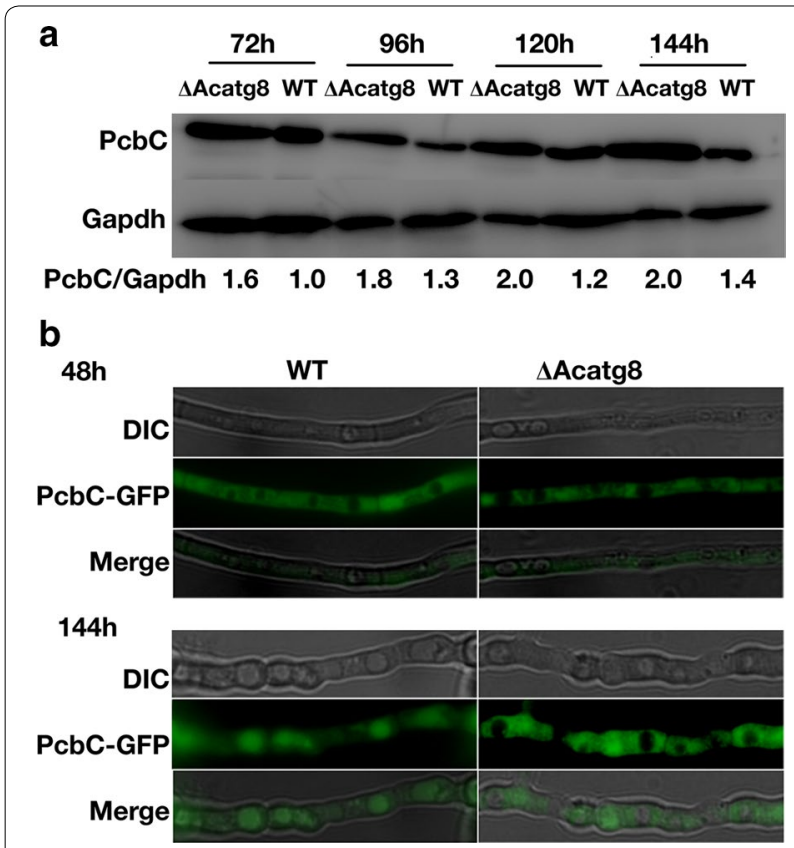

Fig. 6 Detection of $\mathrm{PcbC}$ in WT and $\triangle$ Acatg 8 during fermentation. a Western blot analysis of PcbC in WT and $\triangle A c a t g 8$. Mycelia cultured in the modified MDFA medium for $72,96,120$ and $144 \mathrm{~h}$ were collected. $50 \mu \mathrm{g}$ of total protein was loaded for Western blot analysis with anti-PcbC. Gapdh was used as control. The ratio of PcbC to Gapdh is shown at the bottom. $\mathbf{b} \mathrm{PcbC}$ was detected under fluorescence microscope after 48 and $144 \mathrm{~h}$ fermentation. PcbC in WT and $\triangle$ Acatg 8 were labeled with a green fluorescent protein (GFP)

morphological differentiation. Under starvation condition, the mycelia base cells will degrade their material and transport to the apical cells. These nutrients were used to promote the mycelial growth [46]. This mechanism allows the colony to expand and makes it easier to find usable substances. Like most of filamentous fungi, the disruption mutant of Acatg8 could not grow well under starvation condition due to the autophagic defect, indicating that Acatg8 is necessary for the survival of $A$. chrysogenum under starvation condition.

A large number of accumulated mitochondria were also observed in $\Delta$ Acatg8 at the late stage of fermentation (Additional file 1: Fig. S5). In S. cerevisiae, autophagic defect leads to mitochondrial dysfunction and accumulates excessive ROS [47]. In mammalian cells, excessive ROS will induce the autophagy-mediated cell death [48]. This could be the main reason of $\Delta$ Acatg8 viability reduction since dysfunctional mitochondria produce excessive ROS which impairs fungal survival.

Premature death of $\Delta$ Acatg8 is an intractable problem since it will result in cephalosporin biosynthesis termination, it was also found in the Acatg1 disruption mutant [21]. To solve this problem, inducible expression of Acatg8 under $\mathrm{xyl}^{\mathrm{P}}$ was performed in $\Delta$ Acatg8. Inducible

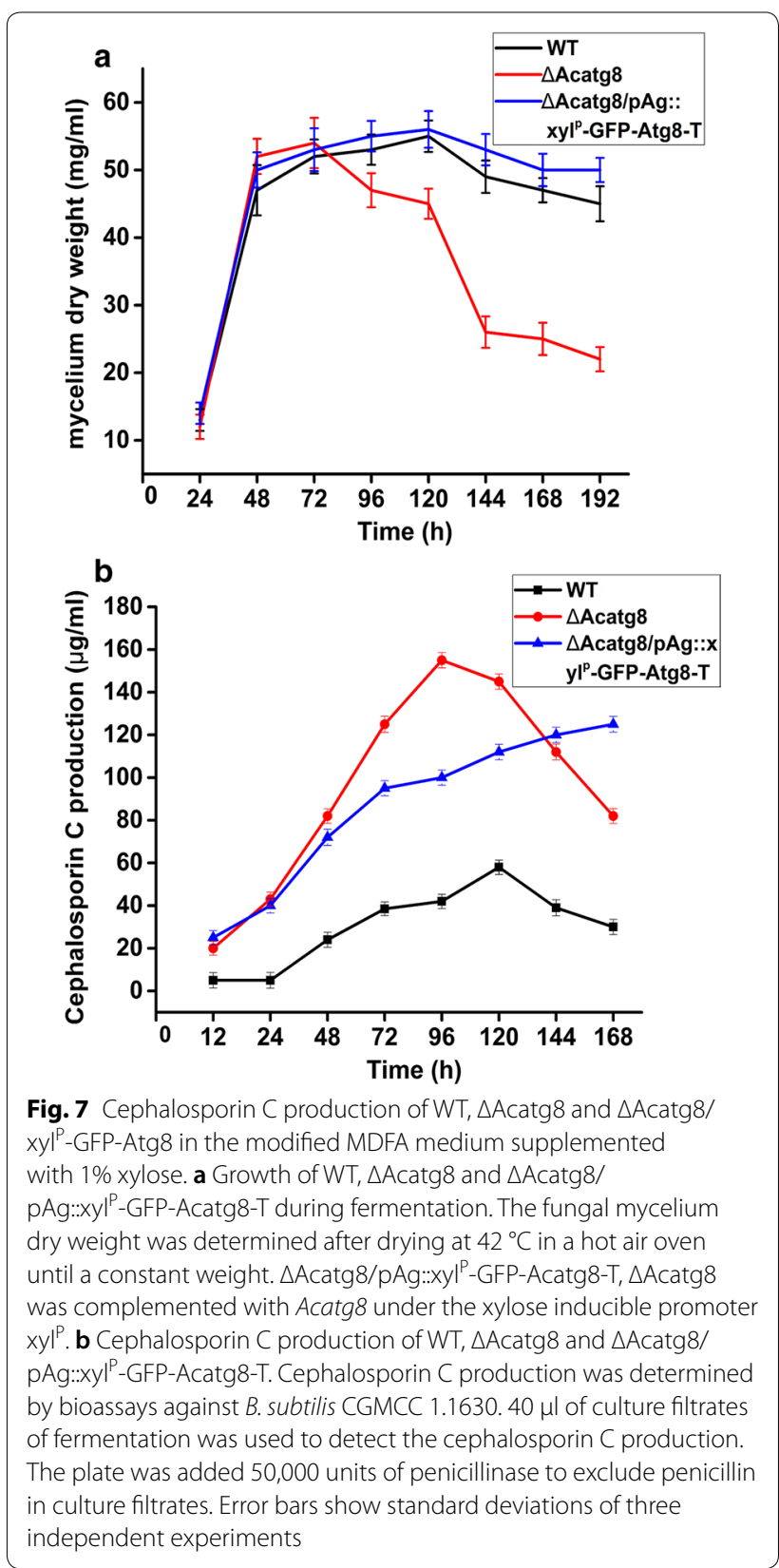

expression of Acatg8 remarkably improved the viability of $\Delta$ Acatg8, especially at the late stage of fermentation when glucose was depleted, while the CPC yield still maintained at a high level. Thus, the inducible expression of autophagy-related genes could be a general method for increasing antibiotic production and maintaining the fungal viability in the autophagic deficiency strains.

\section{Conclusions}

In this study, we identified and characterized an autophagy related gene Acatg8 which could complement the ATG8 disruption mutant ( $\Delta$ atg8) of $S$. cerevisiae. 
AcAtg8 is localized in the cytoplasm and autophagosome of $A$. chrysogenum based on the observation of fluorescently labeled AcAtg8, and the expression of Acatg8 was clearly induced by starvation. Disruption of Acatg8 inhibited the autophagosome formation of $A$. chrysogenum and reduced the fungal conidiation, but increased the CPC production through enhancing the transcription of cephalosporin biosynthetic genes and retaining their products. However, disruption of Acatg8 seriously reduced the fungal viability. Through inducible expression of Acatg8 under the xylose/xylan-inducible promoter $\mathrm{xyl}^{\mathrm{P}}$, the fungal viability was restored while the $\mathrm{CPC}$ production still maintained at a high level. This study provides a promising approach for antibiotic production improvement through modulating the autophagic process of $A$. chrysogenum and extends our understanding of the relationship between secondary metabolite production and fungal autophagy.

\section{Additional file}

Additional file 1: Table S1. Strains and plasmids used in this study. Table S2. Primers used in this study. Fig. S1. Verification of the heterologous complemented strains of $\triangle$ atg 8 by RT-PCR. Fig. S2. Construction of the Acatg8 disruption mutant. Fig. S3. Localization of ACAtg8 during conidial germination of $A$. chrysogenum. Fig. S4. Relative transcriptional level of AcbrlA, AcwetA and AcabaA for conidiation in WT, $\triangle$ Acatg 8 and Acatg8C. Fig. S5. Degradation of mitochondria in WT and $\triangle$ Acatg8. Fig. S6. Complementation of $\triangle$ Acatg 8 with Acatg 8 under control of $x y l^{P}$. Fig. S7. Inducible expression of Acatg8 under control of xyl $\mathrm{P}^{\mathrm{.}}$. Fig. S8. Degradation of peroxisomes in WT and $\triangle$ Acatg8 during fermentation. Fig. S9. Degradation of CefD2 in WT and $\triangle$ Acatg8 during fermentation.

\section{Abbreviations}

WT: the wild-type strain; $\triangle$ Acatg8: the Acatg8 disruption mutant; Acatg8C: the complemented strain of $\triangle A c a t g 8 ; A C V$ : tripeptide $\delta$-(L-a-aminoadipyl)-LCysteinyl-D-valine; IPNS: isopenicillin N synthetase; IPN: isopenicillin N; DAOC: deacetoxycephalosporin C; DAC: deacetylcephalosporin C; ORF: open reading frame; SG-N: nitrogen-starved medium; TEM: transmission electron microscopy; ROS: reactive oxygen species; RFP: red fluorescent protein; GFP: green fluorescent protein; $h p h$ : hygromycin phosphotransferase gene; ble: bleomycin resistance gene; ATMT: Agrobacterium tumefaciens-mediated transformation; CPC: cephalosporin C; MDC: monodansyl cadavarine; PMSF: phenylmethylsulfonyl fluoride.

\section{Authors' contributions}

$\mathrm{HL}, \mathrm{PH}, \mathrm{YW}$ and YP participated in the experiments and analyzed the primary data. $\mathrm{HL}, \mathrm{PH}$ and $\mathrm{YW}$ drafted the manuscript. $\mathrm{HL}$ and $\mathrm{YW}$ carried out all the construction of strains and plasmids used in this study and performed the fermentation and transcription experiments. $\mathrm{PH}$ performed all the fluorescence observation of the fungal cells. YP assisted with the experiment and analysis. GL supervised the whole work and revised the manuscript. All authors read and approved the final manuscript.

\section{Author details}

${ }^{1}$ State Key Laboratory of Mycology, Institute of Microbiology, Chinese Academy of Sciences, Beijing 100101, China. ${ }^{2}$ University of Chinese Academy of Sciences, Beijing 100049, China.

\section{Acknowledgements}

We are grateful to Prof. Juan F. Martín (Universidad de León, Spain) for providing the plasmid pJL43-RNAi and the staffs from Core Facility for Protein Research (Institute of Biophysics, CAS) for helps with transmission electron microscopy analysis. We thank Prof. Wei Li (Institute of Zoology, CAS) for providing Saccharomyces cerevisiae BY4742 and $\triangle$ atg8. We thank Prof. Seogchan Kang (Penn State University, USA) and Prof. Xingzhong Liu (Institute of Microbiology, CAS) for providing plasmid pAg1- $\mathrm{H} 3$.

\section{Competing interests}

The authors declare that they have no competing interests.

\section{Availability of data and materials}

All data for this study are included in this published article and its additional file.

\section{Consent for publication}

The authors are consent for publication.

\section{Ethics approval and consent to participate}

Not applicable.

\section{Funding}

This work was supported by grants from National Natural Science Foundation of China (NSFC, grant numbers 31670091, 31470177 and 31770056).

\section{Publisher's Note}

Springer Nature remains neutral with regard to jurisdictional claims in published maps and institutional affiliations.

Received: 17 October 2017 Accepted: 1 November 2018

Published online: 13 November 2018

\section{References}

1. Avin-Wittenberg T, Michaeli S, Honig A, Galili G. ATI1, a newly identified Atg8-interacting protein, binds two different Atg8 homologs. Plant Signal Behav. 2012;7(6):685-7.

2. Voigt $O$, Herzog B, Jakobshagen A, Pöggeler S. bZIP transcription factor SmJLB1 regulates autophagy-related genes Smatg 8 and Smatg4 and is required for fruiting-body development and vegetative growth in Sordaria macrospora. Fungal Genet Biol. 2013;61:50-60.

3. Levine B, Klionsky DJ. Development by self-digestion: molecular mechanisms and biological functions of autophagy. Dev Cell. 2004;6(4):463-77.

4. Kikuma T, Arioka M, Kitamoto K. Autophagy during conidiation and conidial germination in filamentous fungi. Autophagy. 2007;3(2):128-9.

5. Chen HY, White E. Role of autophagy in cancer prevention. Cancer Prev Res. 2011;4(7):973-83.

6. Madeo F, Tavernarakis N, Kroemer G. Can autophagy promote longevity? Nat Cell Biol. 2010;12(9):842-6.

7. Shpilka T, Welter E, Borovsky N, Amar N, Shimron F, Peleg Y, Elazar Z. Fatty acid synthase is preferentially degraded by autophagy upon nitrogen starvation in yeast. Proc Natl Acad Sci U S A. 2015;112(5):1434-9.

8. Pollack JK, Harris SD, Marten MR. Autophagy in filamentous fungi. Fungal Genet Biol. 2009:46(1):1-8.

9. Nguyen LN, Bormann J, Le GT, Stärkel C, Olsson S, Nosanchuk JD, et al. Autophagy-related lipase FgATG15 of Fusarium graminearum is important for lipid turnover and plant infection. Fungal Genet Biol. 2011;48(3):217-24.

10. Asakura M, Ninomiya S, Sugimoto M, Oku M, Yamashita S, Okuno T, et al. Atg26-mediated pexophagy is required for host invasion by the plant pathogenic fungus Colletotrichum orbiculare. Plant Cell. 2009;21(4):1291-304.

11. Knuppertz L, Hamann A, Pampaloni F, Stelzer E, Osiewacz HD. Identification of autophagy as a longevity-assurance mechanism in the aging model Podospora anserina. Autophagy. 2014;10(5):822-34. 
12. Kikuma T, Ohneda M, Arioka M, Kitamoto K. Functional analysis of the ATG8 homologue Aoatg8 and role of autophagy in differentiation and germination in Aspergillus oryzae. Eukaryot Cell. 2006;5(8):1328-36.

13. Yoon J, Kikuma T, Maruyama J, Kitamoto K. Enhanced production of bovine chymosin by autophagy deficiency in the filamentous fungus Aspergillus oryzae. PLOS ONE. 2013;8(4):e62512.

14. Voigt O, Pöggeler S. Autophagy genes Smatg8 and Smatg4 are required for fruiting-body development, vegetative growth and ascospore germination in the filamentous ascomycete Sordaria macrospora. Autophagy. 2013;9(1):33-49.

15. Bartoszewska M, Kiel JA, Bovenberg RA, Veenhuis M, van der Klei IJ. Autophagy deficiency promotes beta-lactam production in Penicillium chrysogenum. Appl Environ Microbiol. 2011;77(4):1413-22.

16. Liras P, Martín JF. Gene clusters for beta-lactam antibiotics and control of their expression: why have clusters evolved, and from where did they originate? Int Microbiol. 2006;9(1):9-19.

17. Martín JF, Demain AL. Unraveling the methionine-cephalosporin puzzle in Acremonium chrysogenum. Trends Biotechnol. 2002;20(12):502-7.

18. Tollnick C, Seidel G, Beyer M, Schügerl K. Investigations of the production of cephalosporin C by Acremonium chrysogenum. Adv Biochem Eng Biotechnol. 2004;86:1-45.

19. Ruiz B, Chávez A, Forero A, García-Huante Y, Romero A, Sánchez M, et al. Production of microbial secondary metabolites: regulation by the carbon source. Crit Rev Microbiol. 2010;36(2):146-67.

20. Niu G, Zheng J, Tan H. Biosynthesis and combinatorial biosynthesis of antifungal nucleoside antibiotics. Sci China Life Sci. 2017;60(9):939-47.

21. Wang H, Pan Y, Hu P, Zhu Y, Li J, Jiang X, Liu G. The autophagy-related gene Acatg1 is involved in conidiation and cephalosporin production in Acremonium chrysogenum. Fungal Genet Biol. 2014;69:65-74.

22. Liu J, Hao T, Hu P, Pan Y, Jiang X, Liu G. Functional analysis of the selective autophagy related gene Acatg11 in Acremonium chrysogenum. Fungal Genet Biol. 2017;107:67-76.

23. Long L, Wang Y, Yang J, Xu X, Liu G. A septation related gene AcsepH in Acremonium chrysogenum is involved in the cellular differentiation and cephalosporin production. Fungal Genet Biol. 2013;50:11-20.

24. Guan F, Pan Y, Li J, Liu G. A GATA-type transcription factor AcAREB for nitrogen metabolism is involved in regulation of cephalosporin biosynthesis in Acremonium chrysogenum. Sci China Life Sci. 2017;60(9):958-67.

25. Liu P, Zhu H, Zheng G, Jiang W, Lu Y. Metabolic engineering of Streptomyces coelicolor for enhanced prodigiosins (RED) production. Sci China Life Sci. 2017;60:948-57.

26. Zhang $N$, Chen $Y$, Jiang $R$, Li E, Chen $X, X i$, et al. PARP and RIP 1 are required for autophagy induced by $11^{\prime}$-deoxyverticillin $A$, which precedes caspase-dependent apoptosis. Autophagy. 2011;7:598-612.

27. Hu P, Wang Y, Zhou J, Pan Y, Liu G. AcstuA, which encodes an APSES transcription regulator, is involved in conidiation, cephalosporin biosynthesis and cell wall integrity of Acremonium chrysogenum. Fungal Genet Biol. 2015;83:26-40.

28. Zhuo J, Ma B, Xu J, Hu W, Zhang J, Tan H, Tian Y. Reconstruction of a hybrid nucleoside antibiotic gene cluster based on scarless modification of large DNA fragments. Sci China Life Sci. 2017;60(9):968-79.

29. Matthias J, Mebling S, Eichinger L. The two Dictyostelium autophagy eight proteins, ATG8a and ATG8b, associate with the autophagosome in succession. Eur J Cell Biol. 2016;95(1):15-25.
30. Hirata E, Ohya Y, Suzuki K. Atg4 plays an important role in efficient expansion of autophagic isolation membranes by cleaving lipidated Atg8 in Saccharomyces cerevisiae. PLoS ONE. 2017;12(7):e0181047.

31. Deng YZ, Ramos-Pamplona M, Naqvi NI. Autophagy-assisted glycogen catabolism regulates asexual differentiation in Magnaporthe oryzae. Autophagy. 2009;5(1):33-43

32. Blatzer M, Gsaller F, Abt B, Schrettl M, Specht T, Haas H. An endogenous promoter for conditional gene expression in Acremonium chrysogenum: the xylan and xylose inducible promoter xyl1(P.). J Biotechnol. 2014;169:82-6.

33. Voigt $\mathrm{O}$, Pöggeler S. Self-eating to grow and kill: autophagy in filamentous ascomycetes. Appl Microbiol Biotechnol. 2013;97(21):9277-90.

34. Kim JH, Lim JS, Kim CH, Kim SW. Morphology and kinetics studies on cephalosporin C production by Cephalosporium acremonium M25 in a 30-l bioreactor using a mixture of inocula. Lett Appl Microbiol. 2005:40(5):307-11.

35. Queener SW, Ellis LF. Differentition of mutants of Cephalosporium acremonium in complex medium: the formation of unicellular arthrospores and their germination. Can J Microbiol. 1975;21(12):1981-96.

36. StehlikT, Sandrock B, Ast J, Freitag J. Fungal peroxisomes as biosynthetic organelles. Curr Opin Microbiol. 2014;22:8-14.

37. Jedlitschky G, Huber M, Völkl A, Müller M, Leier I, Müller J, et al. Peroxisomal degradation of leukotrienes by beta-oxidation from the omega-end. J Biol Chem. 1991;266(36):24763-72.

38. Bartoszewska M, Opaliński L, Veenhuis M, van der Klei IJ. The significance of peroxisomes in secondary metabolite biosynthesis in filamentous fungi. Biotechnol Lett. 2011;33(10):1921-31.

39. van den Berg MA, Albang R, Albermann K, Badger JH, Daran JM, Driessen AJ, et al. Genome sequencing and analysis of the filamentous fungus Penicillium chrysogenum. Nat Biotechnol. 2008;26(10):1161-8.

40. Opaliński Ł, Kiel JA, Williams C, Veenhuis M, van der Klei IJ. Membrane curvature during peroxisome fission requires Pex11. EMBO J. 2011;30(1):5-16.

41. Martín JF, Ullán RV, García-Estrada C. Role of peroxisomes in the biosynthesis and secretion of $\beta$-lactams and other secondary metabolites. J Ind Microbiol Biotechnol. 2012:39(3):367-82.

42. Mizushima N. The pleiotropic role of autophagy: from protein metabolism to bactericide. Cell Death Differ. 2005;12:1535-41.

43. Yorimitsu T, Klionsky DJ. Autophagy: molecular machinery for self-eating. Cell Death Differ. 2005;12:1542-52.

44. Lulloff SJ, Hahn BL, Sohnle PG. Fungal susceptibility to zinc deprivation. J Lab Clin Med. 2004;144(4):208-14.

45. Tsukada M, Ohsumi Y. Isolation and characterization of autophagydefective mutants of Saccharomyces cerevisiae. FEBS Lett. 1993;333(1-2):169-74.

46. Glass NL, Jacobson DJ, Shiu PK. The genetics of hyphal fusion and vegetative incompatibility in filamentous ascomycete fungi. Annu Rev Genet. 2000;34:165-86.

47. Suzuki SW, Onodera J, Ohsumi Y. Starvation induced cell death in autophagy-defective yeast mutants is caused by mitochondria dysfunction. PLOS ONE. 2011;6(2):e17412.

48. Kim Y, Kim YS, Kim DE, Lee JS, Song JH, Kim HG, et al. BIX-01294 induces autophagy-associated cell death via EHMT2/G9a dysfunction and intracellular reactive oxygen species production. Autophagy. 2013;9(12):2126-39.

Ready to submit your research? Choose BMC and benefit from

- fast, convenient online submission

- thorough peer review by experienced researchers in your field

- rapid publication on acceptance

- support for research data, including large and complex data types

- gold Open Access which fosters wider collaboration and increased citations

- maximum visibility for your research: over 100M website views per year

At BMC, research is always in progress.

Learn more biomedcentral.com/submissions 